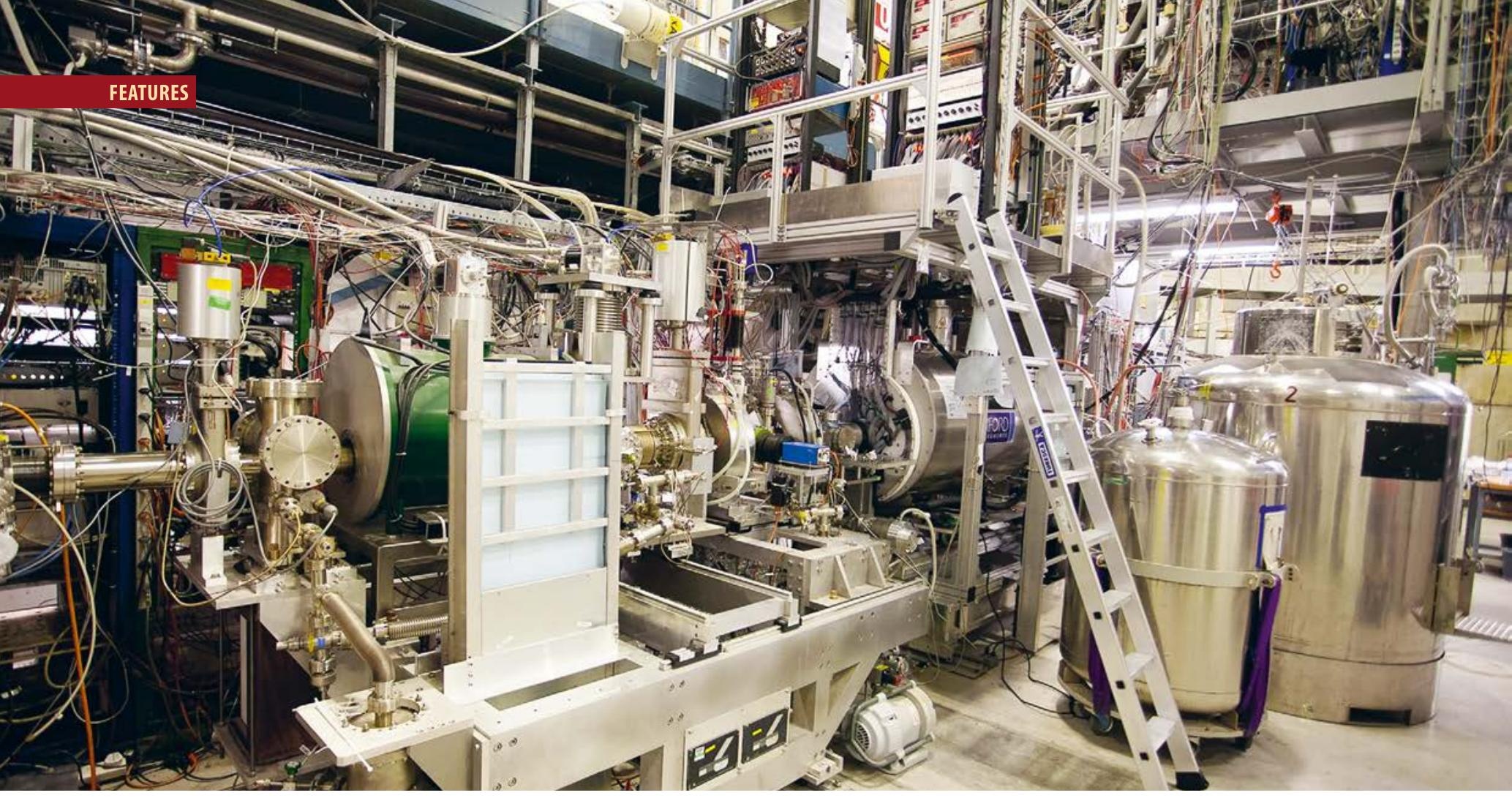

\title{
SPECTROSCOPY OF ANTIHYDROGEN
}

niels Madsen - Swansea University, United Kingdom - DOI: https://doi.org/10.1051/epn/2021404

\section{In 2017 the first observation of an optical transition in an anti-atom was announced by the ALPHA collaboration. This marked a new era in using precision measurements to help unravel one of the most profound questions of modern physics; why the Universe is predominantly made of matter.}

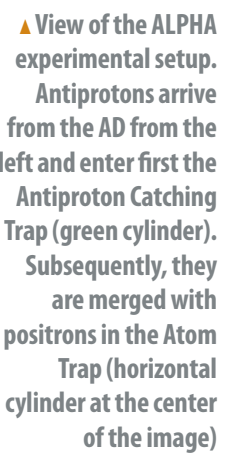

of the image)

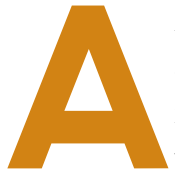

ntihydrogen, the bound state of a positron and an antiproton, was first made at CERN in 1995. It's a testament to the difficulty of this task that the constituents, the positron and the antiproton, were discovered in 1933 and 1955 respectively [1]. The intense interest in antihydrogen reflects the central role hydrogen has played in the development of quantum physics, from Fraunhofer lines in the solar spectrum, over the Balmer series, to the Lamb shift, hydrogen has, due to its simple structure, played a key role in developing a quantum mechanical understanding of the microscopic world. Hydrogen is thus the best understood atom in the periodic table and can be described theoretically from first principles. At the same time, antimatter, or more precisely, the virtual absence of antimatter in the Universe remains an unresolved asymmetry. The famous Sakharov conditions for an asymmetric universe remain a guide for searches, but so far the asymmetries discovered do not lead us to the Universe we observe.
Hydrogen has also helped drive another development, that of precision spectroscopy, and in particular precision laser spectroscopy, where the current record is a measurement of the ground to first excited state (1S-2S) transition to about 15 decimal places of precision [2]. This sort of measurement (though not in hydrogen) is the cornerstone of optical atomic clocks, devices that have recently reached a staggering 18 digits of precision [3]. The technology that has ensued, in particular, the frequency comb developed by Hänsch and Hall [4], has allowed absolute frequency measurements with a significantly more manageable setup than in the past.

To study antihydrogen it needs to be synthesized. Antiprotons are sourced by capturing antiprotons produced in high energy collisions and positrons typically from a radioactive source. These particles are then often manipulated and merged in Penning traps to form antihydrogen that can be trapped using a magnetic minimum trap. An example of the central part of the ALPHA 
experiment to synthesize, trap and study antihydrogen is shown in figure 1. With this state-of-the art apparatus the ALPHA experiment captures about 20 antihydrogen atoms every four minutes and have accumulated more than 1000 atoms in the magnetic trap thus opening the door to very precise scrutiny of the anti-atoms $[1,5]$.

Combining advances in precision spectroscopy with advances in antihydrogen synthesis thus holds the promise for extremely accurate comparisons of matter and antimatter that may lead to the discovery of a difference between them that has so far eluded other approaches.

\section{Spectroscopy}

The headline transition sought in hydrogen and antihydrogen is the dipole forbidden $1 S-2 S$ transition already mentioned. As it is dipole forbidden, two photons need to be emitted for a decay and the lifetime is thus very long (about $1 / 8$ of a second), and the linewidth correspondingly narrow. This narrow linewidth and the elimination of the first order Doppler shift by using two photons for excitation is key to the spectacular precision of the hydrogen measurement, and thereby its promise of the most precise comparison of hydrogen and antihydrogen.

However, when looking for symmetry violations, it must be kept in mind that different transitions depend differently on the underlying interactions. Furthermore, the (anti)proton charge radius influences the energy levels (see e.g. The Proton Radius Puzzle [6]). There is therefore ample motivation to study several other transitions amongst which the most prominent are perhaps the ground state hyperfine splitting and the $2 S$ Lamb shift.

In contrast to hydrogen, which can be difficult to detect, antihydrogen annihilates on impact with matter, and the $\sim 2 \mathrm{GeV}$ of energy released from the antiproton annihilation, mostly in the form of pions, is easily detectable, such that, under the right circumstances, the loss of a single antihydrogen atom can be observed. This sensitivity allowed the first trapping to be confirmed and has also served as the workhorse for all subsequent measurements. The initial trapping in 2010 and subsequent improvements has allowed the ALPHA experiment to measure the hyperfine splitting, the 1 S- 2 S transition, and the $1 \mathrm{~S}-2 \mathrm{P}$ transition, with the latter recently allowing the first laser-cooling of antihydrogen [7]. The measurement of the $1 S-2 S$ two photon transition stands out by achieving a record (for antihydrogen) precision of $2 \times 10^{-12}$. The signal in these various measurements is based on intentional annihilation of antiprotons, achieved either by inducing a spin flip of the positrons or photoionization of the anti-atom. The precision of the $1 \mathrm{~S}-2 \mathrm{~S}$ measurement is limited by a combination of issues, prominently featuring the transit-time of antihydrogen atoms going through the laser beam. Both a larger laser beam and colder antihydrogen may improve this by narrowing the line width, the promise of the latter recently demonstrated by laser-cooling the antihydrogen atoms. Additionally, improvements in metrology (absolute frequency referencing) are being implemented by adding an active hydrogen maser and a Cs-fountain clock to expedite progress towards the same precision as in hydrogen.

\section{Comparisons with Hydrogen}

The beauty of antihydrogen as a probe for fundamental tests is that it is, at least in principle, possible to make completely model independent comparisons. However, one caveat of measurements on trapped antihydrogen is the presence of the magnetic fields used for trapping that induce significant shifts in the atomic transitions. Ultimately the $1 \mathrm{~S}-2 \mathrm{~S}$ measurement discussed in detail above, as well as other measurements, both planned and done, need to be compared directly to those in hydrogen. However, to truly do that in the most model $\bullet \bullet$

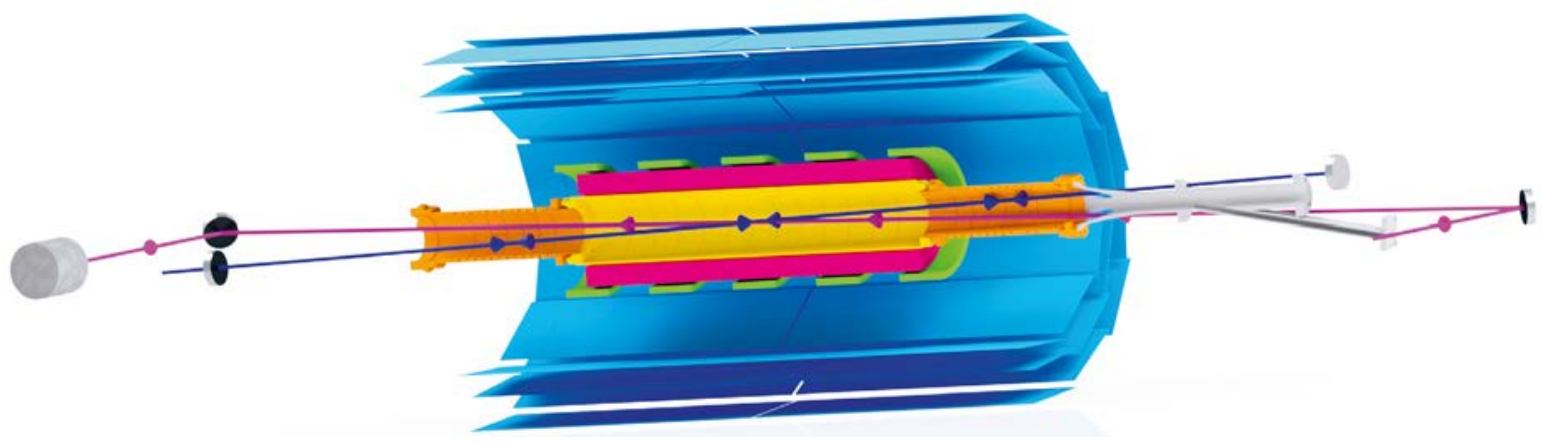

$\triangle$ FIG. 1: Cut-out view of the central ALPHA antihydrogen apparatus. The green cylinders and the long purple cylinder mark the short solenoids and the octupole used for creating the 3D magnetic field strength minimum that serves to trap the antihydrogen atoms. The yellow cylinders are the central Penning trap electrodes that also surround the magnetic trap for antihydrogen synthesis. The sections with orange electrodes are used for preparation of positrons and antiprotons. An axial magnetic field of $1 T$ to complete the Penning trap is supplied by a large solenoid surrounding the whole structure (not shown). Trapped antihydrogen can occupy a volume of around $1 / 2$ liter. The blue laser-beam is the retroreflected $243 \mathrm{~nm}$ laser for $1 \mathrm{~S}-2 \mathrm{~S}$ two-photon excitation (it circulates in a cryogenic build-up cavity), whereas the purple (single pass) laser is the $121.6 \mathrm{~nm}$ laser for 1S-2P excitation and laser-cooling. The grey structures on the left allow microwaves to be injected for inducing hyperfine transitions. The blue panels are the main silicon wafers of the annihilation detector. 
- independent way, a measurement of hydrogen needs to be carried out in the same apparatus, using the same methods, preferably at (at least on average) the same time and also preferably at (near) zero electric and magnetic fields. We're still some way from that. ASACUSA recently made a first step in this direction by using the apparatus they have built for antihydrogen hyperfine measurements to make a hydrogen measurement [8]. The equivalent antihydrogen measurement is pending their ability to make a beam of ground state atoms, but the potential is for a field-free measurement as the antihydrogen is extracted from the synthesis region that necessarily has strong magnetic fields.

For 1S-2S spectroscopy, where significantly more (anti)-atoms are still needed, or at least longer interaction times, the trap still seems the best choice. Thus, antihydrogen compatible methods for loading traps with cold hydrogen must be developed, as must detection methods that do not use annihilations but are compatible with the current setup. The difficulty here is, as illustrated on figure 1 that we only have axial access to the central, cryogenic region, such that efficient photon detection is only possible with in-situ ultra-high vacuum, cryogenic compatible detectors integrated in the cylindrical structure of the Penning-trap electrode stack. However, once developed, such detectors will allow much more sophisticated measurements, also on higher lying states in antihydrogen necessary for the determination of the charge radius of the antiproton and the antimatter Rydberg constant.

\section{Outlook}

Spectroscopy of antihydrogen has come a long way from the early dreams more than 30 years ago, to the accomplishments of the last few years, heralding the most precise measurements of antihydrogen to date. While experimental and systematic challenges remain, the prospect of reaching the same precision as hydrogen now seems within reach for at least the $1 \mathrm{~S}-2 \mathrm{~S}$ transition, as does the prospect of determining the anti-atom Rydberg constant and the antiproton charge radius. Beyond that lies the exciting prospect of utilizing the full potential of the achievements making the comparisons with hydrogen

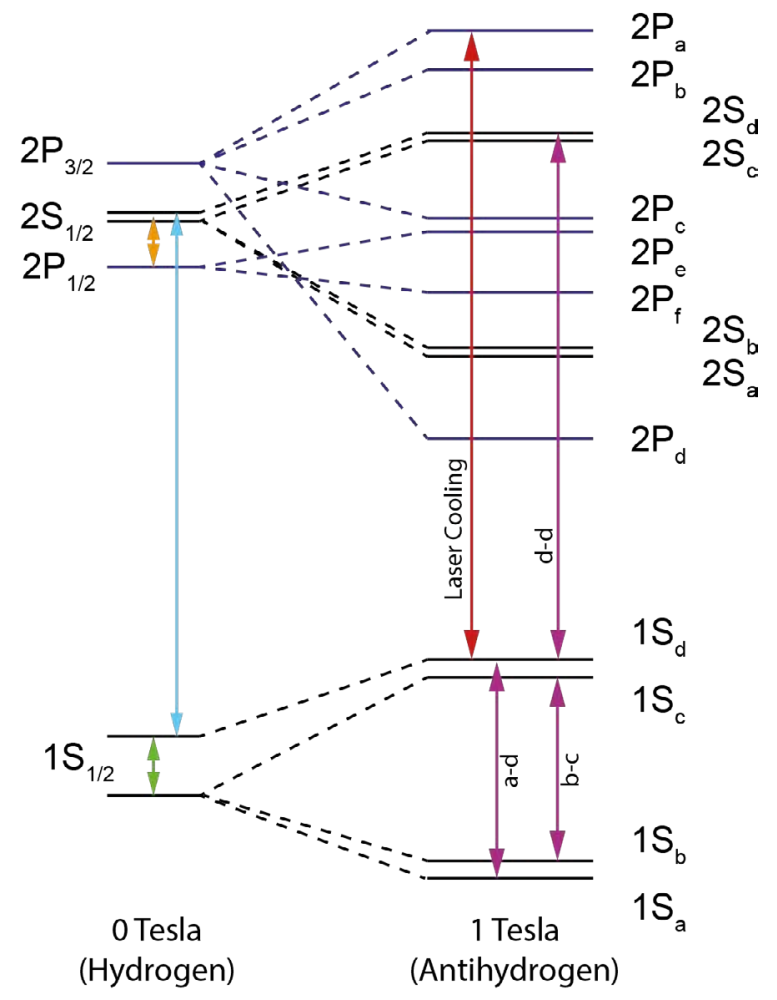

$\triangle$ FIG. 2: Level diagram of (anti)hydrogen in no magnetic field and the 1 Tesla field used in the ALPHA trap. The hyperfine splitting of the 2P levels (in blue) are not shown. Precision measurements in hydrogen have been done near zero magnetic field and we have indicated the most famous splittings : the 1S-2S transition (light blue), the ground state hyperfine splitting (green) and the 2S Lamb shift (orange). For $1 \mathrm{~T}$ (antihydrogen) we have indicated the main precision measurements, the hyperfine transitions in the $1 \mathrm{~S}$ level and the $1 S-2 S$ transition (purple), as well as one of the $1 \mathrm{~S}-2 \mathrm{P}$ transitions, namely the one used for laser-cooling (red).

truly model-independent by doing near-simultaneous measurements in the same apparatus. Antihydrogen spectroscopy is in for an exciting decade, if not more.

\section{About the Author}

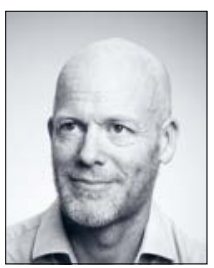

Niels Madsen is professor in experimental physics at Swansea University and deputy spokesperson of ALPHA. He has worked on antihydrogen for 20 years, from the first low energy formation to trapping and spectroscopy.

\section{In contrast to hydrogen, which can be difficult} to detect, antihydrogen annihilates on impact with matter, and the $\sim 2 \mathrm{GeV}$ of energy released from the antiproton annihilation, mostly in the form of pions, is easily detectable, such that, under the right circumstance, the loss of a single antihydrogen atom can be observed.
References

[1] W. A. Bertsche, E. Butler, M. Charlton and N. Madsen, J. Phys. B 48, 232001 (2015)

[2] C. Parthey et al., Phys. Rev. Lett. 107, 203001 (2011)

[3] K. Beloy et al., Nature 591, 564 (2021)

[4] T. Fortier and E. Bauman, Comm. Phys. 2, 153 (2019)

[5] Phil. Trans. Roy. Soc. A 376 (2018) Issue 2116.

[6] W. Ubachs, Science 370, 1033 (2020)

[7] For recent ALPHA publications visit https://alpha.web.cern.ch/

[8] For recent ASACUSA publications visit https://asacusa.web.cern.ch/ 


\section{PFEIFFER VACUUM}

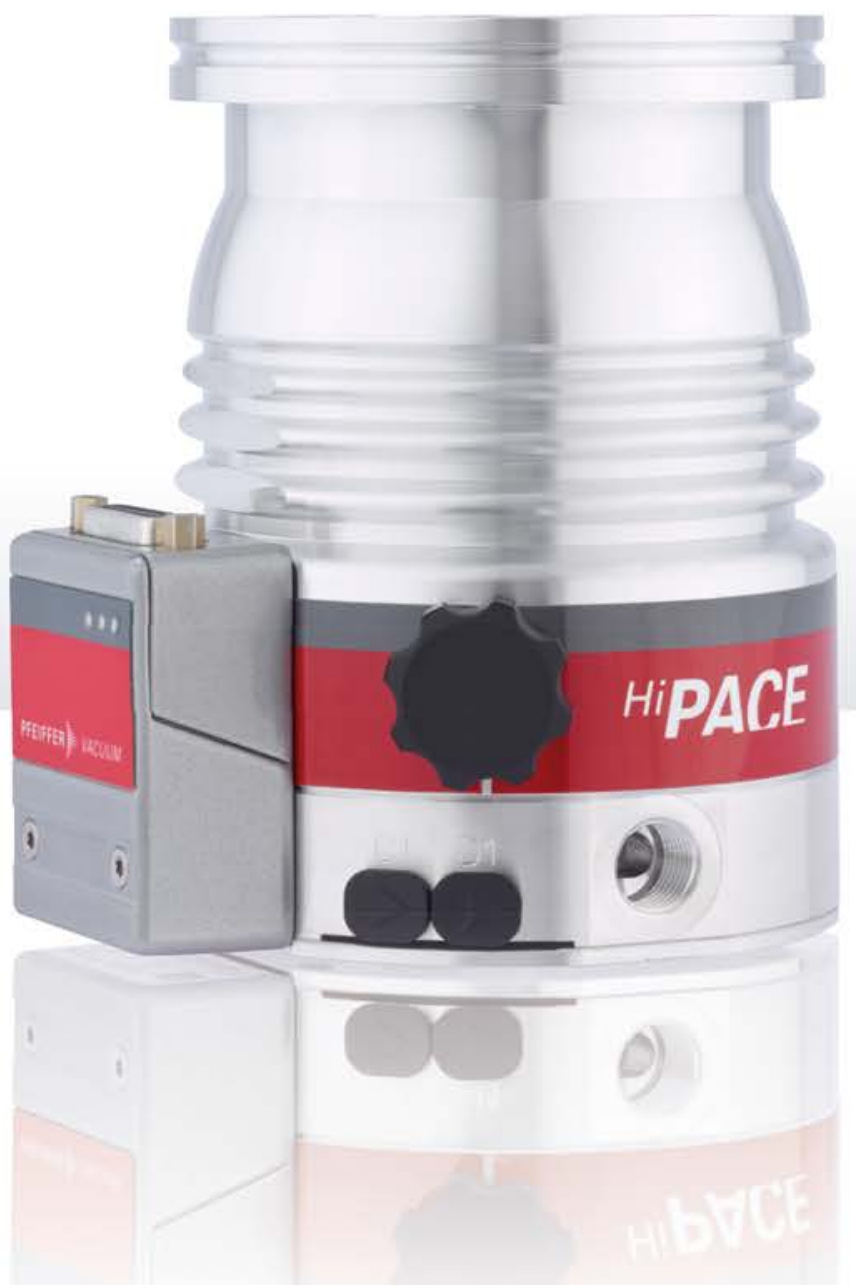

HPace 80 Neo

The hybrid bearing vacuum pump with patented Laser Balancing ${ }^{\mathrm{TM}}$ technology

- Best combination of size and performance due to intelligent rotor temperature measurement

- Lowest vibration level in the market due to Laser Balancing ${ }^{\mathrm{TM}}$ technology

- High compression ratio for light gases

- Oil lubrication with synthetic filtering media to maximize bearing lifetime

Are you looking for a perfect vacuum solution? Please contact us:

Pfeiffer Vacuum GmbH - Germany · T +49 6441 802-0 - www.pfeiffer-vacuum.com 